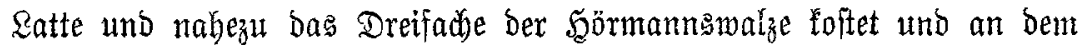
mich meiter interefifiert hat, baj es, wie auf erfter Seite des Profpettes

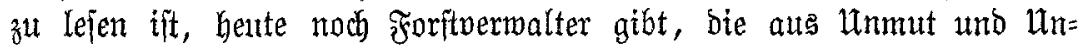
zufriedenheit über ungenügende Reiftungen ber Ëblinger = Ratte wieder zur alten Sondfant zuräd

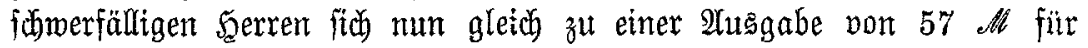

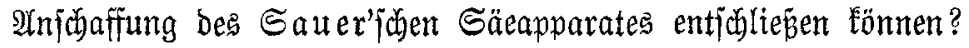

YYs ein neuer uns zur Erprobung übergebener Âpparat zum 3mecf rajber und billiger şerjobitung jei nun bejdrieben

3.

\title{
Dive lleximulplatte
}

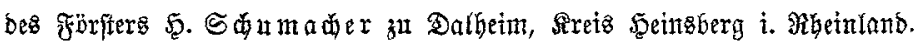

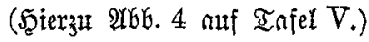

Die "Serjohulplatte" ift aus einem meterlangen, $24 \mathrm{~cm}$ bretten Stüd ftarfen verzintten Eifenblechs bergeftellt, das zu einer $12 \mathrm{~cm}$ breiten Slatte zujammengebogen itt; bie itbereinanber liegenben offenen Seiten enthalten in je $5 \mathrm{~cm}$ Entfernung Eleine Freisförmige 2 lugjofnitte unb find burch Nieten verbunden, nadbom vorber zwijhen biejelbent ein $3 \mathrm{~cm}$ breiter ftarfer Gammiftreifen fo eingebraht morden, bas berfelbe etwa

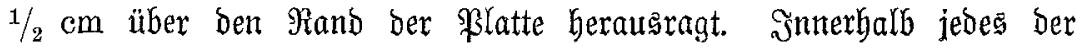

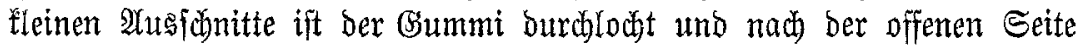

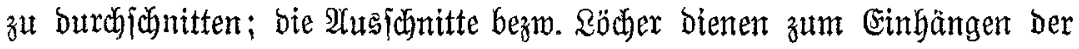

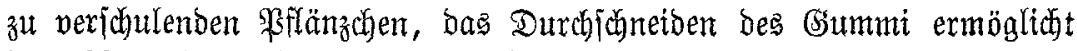
bas פSegnehnen ber \$latte nach erfolgtem Feftpflanzen ber lesteren.

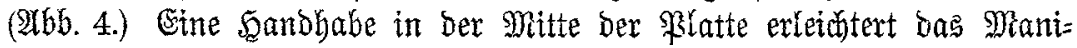
pulieren mit Derjelben.

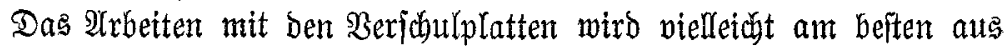

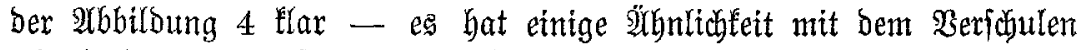

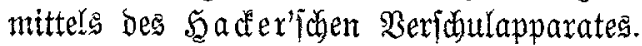

Die Serjobulung erfolgt in sänoem von 3, 4, $5 \mathrm{~m}$ Breite; rechts

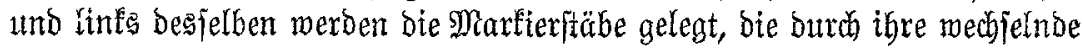
Jarbe (grün=meí) Die (Entfernung ber Ffflanzreihen $(20 \mathrm{~cm})$ geben, eine Seine wirb von Marfierftab zu Martierftab geipannt und mit einer ftarfent

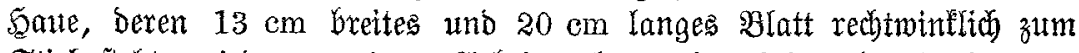
Stiel fteht, wird pon einem Qrrbeiter längs Der \&eine ein Braben nit

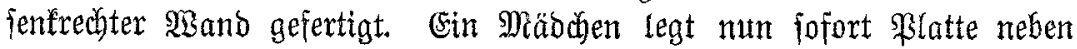
Flatte, jo baj beren Gsummirand etwa $1 \mathrm{~cm}$ vom Fand bes Grabens liegt und bängt bie Pflanzen in bie Röber im Gummi, fo bap beren 
Matrzeln ber Brabenwanb anliegen. F̧ierauf wirb bie jeitlich bes Sräbchens wallartig aufgebäufte Eroe mit Silfe eines bejonderg fon= ftruierten Erbanjibiebers - im Sandboben auch mit einer $40 \mathrm{~cm}$ langen Satte, in Rehmboden mit 4-5zähnigen Düngergabeln - an bie $\mathfrak{\text { Burzeln }}$

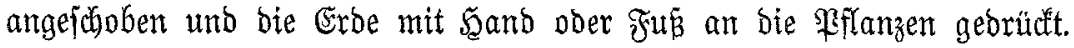
Die Flatten merben nach Dem Einpflanzen vorifibtig weggezogen, wobet bie Pfflanzen von felbft burd) bie Einfohnitte ats bem çummi fpringen, und bie leeren \$ilatten etwas zurüfolelegt. Sierauf wirb ber Boben neben Der \$ffanzreife auf $20 \mathrm{~cm}$ Breite geebnet, bie Reine neu gezogen und bie nächite Reihe begonnen.

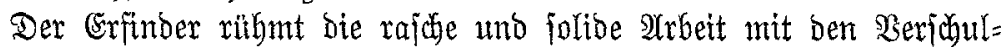
platten, befonbers bei Serichulung 2-3jähriger Fibhten, und foll es

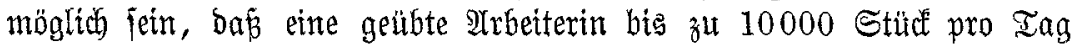
verfichult. Da eine \$latte 20 \$flanzen fabt, jo wurbe bies einem sin=

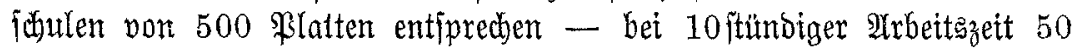
Platten pro Stumbe oder nahezul einer slatte pro Mimute; mit Braben= anjertigung, sinbängen ber Fffanzen, Feetpflanzen derjelben und cin: ebnen bes Bobens mürbe bies eine ganz auperorbentlicte seiftung jein,

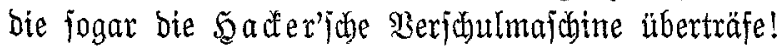

Ein vergleichender Berjuch, ber im hiefigen Fonitgarten mit Den

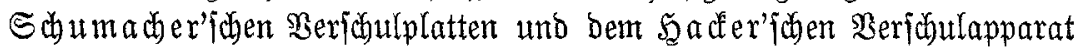
(nicht ber Maf(bine) angeitellt und bei welchent 2 trbeiter verwendet wurben,

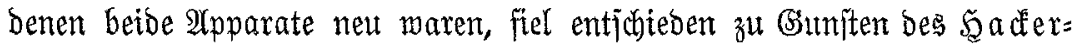

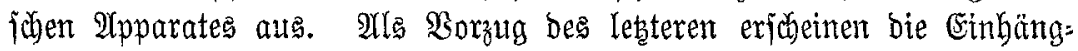
latten, welche ein $\mathfrak{B} e r i b u l e n$ in jedem beliebigen 2 Ubitano $(21 / 2,5,71 / 2$, $10 \mathrm{~cm}$ ) ermöglichen, bei welchen ferner bas Eingängen Der Pffanzen jeitens Der 2lrbeiterinnen mit Şilfe bex einfachen zum 2lpparat gebörigen Salter fteheno uno alfo viel bequemer exfolgt, uno enolich bas $\mathfrak{B}$ seg= nefmen ber \&aiten fehr einfach und ficher gejofiebt, mährend bei ben

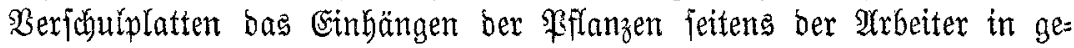
bücơter Stellung ftattfinden mǘ, beim $\mathfrak{B e g n e b m e n ~ b e r ~ \Re l a t t e n ~ a b e r ~}$ nicht felten \$fflänzchen iun Grummi bängen bleiben uno mebr ober wentger aus bem Boben gezogen werden.

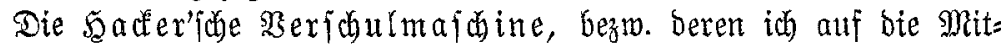

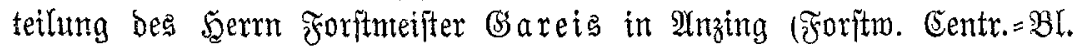
1903, S. 233) permeife, uno bie fid immer weitere Berbreitung erringt - fo murben beren für bas Grobhergogtum Sefjen nibt wentger als

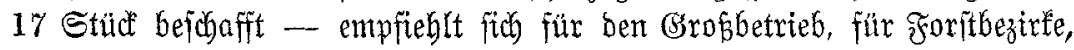

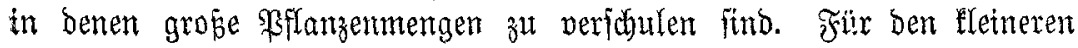




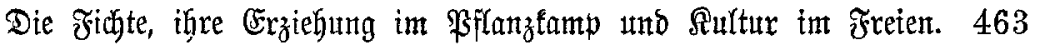

Betrieb, einen Bedarf von $30-40$ Taufend verfdulter \$ffanzen, mödte

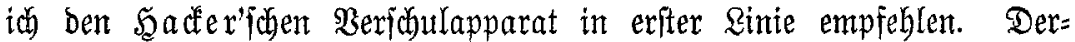

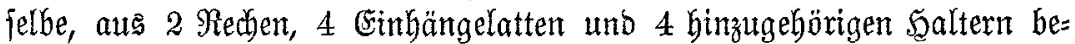

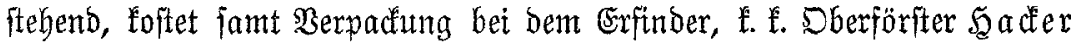

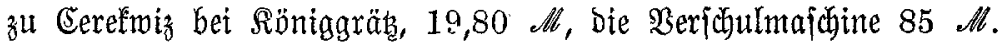

Die Berichulplatten, beren seifungşähyigfeit bei entiprechender übung ber Yrbeiter id auf Grumb ber wom Erfinder mitgeteilten

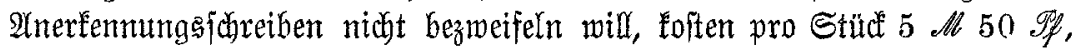

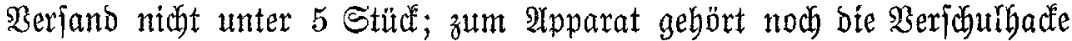
(3 Mi), Martertiäbe, Reine und $\mathfrak{A n j}$ diteber.

Sch möbte übrigens bei biejer Belegenheit bemerten, baj nad)

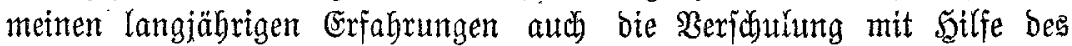

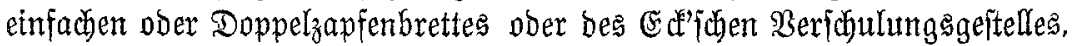
wie joldse in meiner "Pllanzenzutht im SBalbe" bejwrieben ift, jebr gute

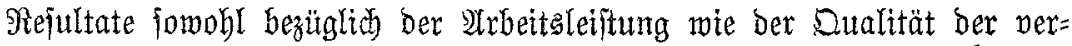
fobulten Pflanzen ergibt, wovon ich bet ber eingangs ermäbnten " Probe im Froritgarten bie ßollegen überzengen fonnte. Bebingung ift nur bie Serftellung genitgend tiefer und weiter Sfflanzlöber burd ausreichent grop̉e und lange Bapfen, fowie Berwendung geidulter 2robeiter, bie ein

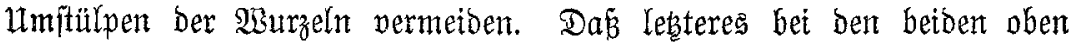
gejefilderten Itpparaten abjolut vermieben wirb, ift als ein entidgiebener গ̧orz̆tg berjelben zu betrachten.

Die Berioulung fräftiger einjäbriger fribten balte ich für bas 3meckmäpigigite, ebenio eine etwas enge \$erichulung - $5 \mathrm{~cm}$ Fflanzen= abitand in $10-12 \mathrm{~cm}$ entfernten Sheiken - für bie Erziehung brei=

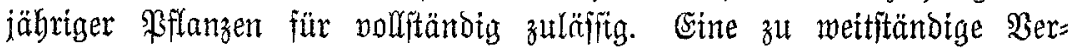
fadulung erböht bie Soiten jebr bebeuteno!

\section{Die fichte}

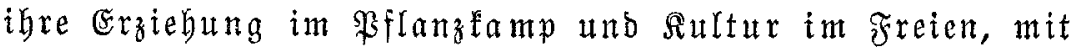
befonderer Berüfifictigung ber in norböftiden Ietl bes Dbenwalbes gelegenen Dberföriterei Bielbrunn.

Bon Sberförfter Mi, von tiblagger in Eaubentad a. MR.

Es jollen vorker furrz bie Grü̈nde hervorgehoben werben, welche

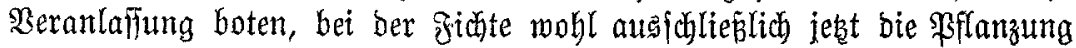
in Ânmendung zut bringen.

Miöge bie Saat meldent Mamen immer führen, faft ftets ift fie zlt ichütter ober fie ift zat bicht; - betbes find gleich grope Febler! Selten 